\title{
Approximation on Manifold
}

\author{
YU.K.DEM'YANOVICH \\ St. Petersburg State University \\ Department of Parallel Algorithms \\ 7/9 Universitetskaya nab. \\ St. Petersburg, RUSSIA \\ y.demjanovich@spbu.ru
}

\begin{abstract}
The purpose of this work is to obtain an effective evaluation of the speed of convergence for multidimensional approximations of the functions define on the differential manifold. Two approaches to approximation of functions, which are given on the manifold, are considered. The firs approach is the direct use of the approximation relations for the discussed manifold. The second approach is related to using the atlas of the manifold to utilise a well-designed approximation apparatus on the plane (finit element approximation, etc.). The firs approach is characterized by the independent construction and direct solution of the approximation relations. In this case the approximation relations are considered as a system of linear algebraic equations (with respect to the unknowns basic functions $\omega_{j}(\zeta)$ ). This approach is called direct approximation construction. In the second approach, an approximation on a manifold is induced by the approximations in tangent spaces, for example, the Courant or the Zlamal or the Argyris fla approximations. Here we discuss the Courant fla approximations. In complex cases (in the multidimensional case or for increased requirements of smoothness) the second approach is more convenient. Both approaches require no processes cutting the manifold into a finit number of parts and then gluing the approximations obtained on each of the mentioned parts. This paper contains two examples of Courant type approximations. These approximations illustrate the both approaches mentioned above.
\end{abstract}

Key-Words: approximation, manifold, simplicial subdivision, splines

Received: September 18, 2020. Revised: March 1, 2021. Accepted: March 17, 2021. Published: March 29, 2021.

\section{Introduction}

The solving of the complicated problems for modeling various phenomena is often connected with the approximate search of functions in regions of very complex configurations Examples of such tasks are the tasks of forecasting climate, weather, geological cataclysms, etc. In the listed tasks, the mentioned areas are a sphere, a ball or a spherical layer. The solution of technological problems are often connected with difficul areas. For example, channels of different shapes are required for the propagation of radiation, and a toroidal structure are required for the $\mathrm{fl} \mathrm{w}$ of elementary particles in a collider, etc.

When buildings and structures are being designed, shells are considered. The last one is a region of definition for various functions. Thus, the problem of approximation of functions with a complexly structured area definitio is highly relevant. Mathematical formulation of the problem is an approximation of functions define on manifolds. Solutions of this problem is necessary when we use the methods of finit elements, grid method, wavelet decomposition complexly structured streams and so on.

In practice calculations are considered the various options of the functions with specifi types of domains (see [1] -[28]).

It is very important to investigate the approximation apparatus for the functions given on the manifold. Some progress in this direction was reached in the last century (see [22] and monograph [1]). The order of the rate of convergence of the proposed results in the approximations being the same as for fla regions. However, the question of efficientl calculating the constants in the estimates of the convergence rate has not been resolved.

In what follows, approximations of functions define on the manifolds are considered in the various aspects: the considered manifold approximation by moving least-squares projection (see [19]), entropy estimation in manifold was studied (see [20]), studied Local linear regression on manifolds (see [21]), local and spline approximations on manifold (see [22] - [23]). In addition, wavelet decomposition of $\mathrm{fl}$ ws associated with a manifold (see [24] - [26]). Some relevant studies can be found in [27] and [28].

The effectiveness of the methods and algorithms consists of the fact that their structure is determined by the structure of the manifold. In the mentioned cases the effectiveness manifests itself in the restructuring 
of the spaces depending on the structure of manifold.

The purpose of this work is to obtain an effective evaluation of the speed of convergence for multidimensional approximations of the functions define on the differential manifold.

Here we consider two approaches to constructing the approximation of functions, given on the manifold. The firs approach is the direct use of the above approximation relations for the discussed manifold, the extraction of these coordinate functions and the constructing approximations by the elements corresponding to the linear space. The second approach is related to using the atlas to connect a well-designed approximation apparatus on the plane (finit element approximation, etc.). Both approaches require no processes cutting the manifold into a finit number of parts and then gluing the approximations obtained on each of the mentioned parts.

The firs approach is characterized by the independent construction and direct solution of the approximation relations. In this case the approximation relations are considered as a system of linear algebraic equations (with respect to unknowns $\omega_{j}(\zeta)$ ). This approach is called direct approximation construction.

In the second approach, an approximation on a manifold is induced by the approximations in tangent spaces, for example, the Courant or the Zlamal or the Argyris fla approximations. Here we discuss the Courant fla approximations (see subsections 4.3 and 4.6) In complex cases (In the multidimensional case or for increased requirements of smoothness) the second approach is more convenient.

\section{Auxiliary Assertion}

Consider a smooth $n$-dimensional (generally speaking, noncompact) manifold $\mathbf{M}$. We introduce a system of differentiable coordinates on $\mathbf{M}$, i.e. a family of open sets $\left\{U_{\zeta}\right\}_{\zeta \in \mathcal{Z}}$ covering $\mathbf{M}$ and such homeomorphisms $\psi_{\zeta}, \psi_{\zeta}: E_{\zeta} \mapsto U_{\zeta}$ of open balls $E_{\zeta}$ of the space $\mathbf{R}^{n}$ that the maps

$$
\psi_{\zeta}^{-1} \psi_{\zeta^{\prime}}: \psi_{\zeta^{\prime}}^{-1}\left(U_{\zeta} \cap U_{\zeta^{\prime}}\right) \mapsto \psi_{\zeta}^{-1}\left(U_{\zeta} \cap U_{\zeta^{\prime}}\right)
$$

(for all those $\zeta, \zeta^{\prime} \in \mathcal{Z}$ for which $U_{\zeta} \cap U_{\zeta^{\prime}} \neq \emptyset$ ) are continuously differentiable (desired number of times); here $\mathcal{Z}$ is a set of indices. Troika $\psi_{\zeta}: E_{\zeta} \mapsto U_{\zeta}$ is called a map, a bunch of $\mathbf{A}=\left\{\psi_{\zeta}: \mathbf{E}_{\zeta} \mapsto \mathbf{U}_{\zeta} \mid \zeta \in\right.$ $\mathcal{Z}\}$ is named an atlas representing the manifold $\mathbf{M}$. Without loss of generality, in what follows we assume that the balls $E_{\zeta}$ are contained in the closed ball $E_{*}$ and they have a center at zero, therefore

$$
\psi_{\zeta}(0)=\zeta .
$$

\subsection{Atlas for sphere}

For example, let $\mathbf{M}$ be $n$-dimensional sphere

$$
\mathbf{M}: \quad(\xi, \xi)=\mathbf{r}^{2} .
$$

Here the scalar product in the space $\mathbf{R}^{\mathbf{n}+\mathbf{1}}$ is denoted by the parentheses. We introduce the norm in the mentioned space by the formula $\|\xi\|=(\xi, \xi)^{1 / 2}$.

Let $L_{\zeta}$ be hyperplane to $\mathbf{M}$ in the point $\zeta \in \mathbf{M}$,

$$
L_{\zeta}: \quad\left(\xi^{\prime \prime}, \zeta\right)=r^{2} .
$$

Let us defin the distance $d$ from the point $\xi$ to the hyperplane (4):

$$
d=r-(\xi, \zeta) r^{-1}
$$

Let the vector $\xi^{\prime}$ such that $\xi^{\prime}+\zeta$ is the orthogonal projection of the point $\xi \in \mathbf{M}$ onto $L_{\zeta}$ ( $\zeta$ lies on the nearest hemisphere),

$$
\xi^{\prime}=\xi+\zeta\left(d r^{-1}-1\right) .
$$

Taking into account $\left\|\xi^{\prime}\right\|^{2}+d^{2}=\|\zeta-\xi\|^{2}$ and (5) we have

$$
\psi_{\zeta}: \quad \xi^{\prime} \mapsto \xi, \quad \xi=\xi^{\prime}+\zeta\left(1-\left\|\xi^{\prime}\right\|^{2} r^{-2}\right)^{1 / 2} .
$$

On the other hand, substituting (5) into (6) we obtain

$$
\psi_{\zeta}^{-1}: \quad \xi \mapsto \xi^{\prime}, \quad \xi^{\prime}=\xi-(\xi, \zeta) \zeta r^{-2} .
$$

Transformation (7) - (8) is called an orthogonal projection to a tangent plane.

Lemma 1. 1 . If $\left\|\xi_{i}^{\prime}\right\| \leq q r, q<1, i=1,2$, then a value $M(q)$ exists such that

$$
\left\|\psi_{\zeta}\left(\xi_{1}^{\prime}\right)-\psi_{\zeta}\left(\xi_{2}^{\prime}\right)\right\| \leq M(q)\left\|\xi_{1}^{\prime}-\xi_{2}^{\prime}\right\| .
$$

2. If $\xi_{i} \in \mathbf{M}, i=1,2$, then

$$
\left\|\psi_{\zeta}^{-1}\left(\xi_{1}\right)-\psi_{\zeta}^{-1}\left(\xi_{2}\right)\right\| \leq 2\left\|\xi_{1}-\xi_{2}\right\| .
$$

Proof is elementary. We do not present it.

In the one-dimensional case we introduce the angular variable $\varphi$ on the circumference M. Using (8) and taking into account the orthogonality $\xi^{\prime}$ and $\xi$ we fin $\left\|\xi^{\prime}\right\|^{2}=r^{2}-(\xi, \zeta)^{2}$, hence

$$
\psi_{\zeta}^{-1}: \quad s_{\xi^{\prime}}=r \sin \left(\varphi_{\xi}-\varphi_{\zeta}\right),
$$

where $s_{\xi^{\prime}}$ is a natural parameter on the tangent line $L_{\zeta}$, which increases together with the increase of angles on M. $\varphi_{\xi}$ and $\varphi_{\zeta}$ are angular values of the angle variable corresponding to points $\xi$ and $\zeta$ on $\mathbf{M}$. 


\subsection{On invariance of a polynomial space}

In what follows, $\alpha$ and $\beta$ will denote finite dimensional integer vectors with nonnegative components, and the component number will be marked with an index below:

$$
\begin{gathered}
\alpha=\left(\alpha_{1}, \ldots, \alpha_{n+1}\right), \beta=\left(\beta_{1}, \ldots, \beta_{n+1}\right), \\
|\alpha|=\sum_{i=1}^{n+1} \alpha_{i}, \quad|\beta|=\sum_{i=1}^{n+1} \beta_{i} .
\end{gathered}
$$

Let the system $\mathbf{R}^{\mathbf{n}+\mathbf{1}}$ be fi ed in the space coordinates. The components of the vector $\xi \in \mathbf{R}^{\mathbf{n}+\mathbf{1}}$ in the system will be numbered with a superscript enclosed in the boxes, $\xi=\left(\xi^{(1)}, \xi^{(2)}, \ldots, \xi^{(n+1)}\right)$. For vector $\beta=\left(\beta_{1}, \ldots, \beta_{n+1}\right)$ we put

$$
\xi^{\beta}=\left(\xi^{(1)}\right)^{\beta_{1}}\left(\xi^{(2)}\right)^{\beta_{2}} \ldots\left(\xi^{(n+1)}\right)^{\beta_{n+1}},
$$

assuming that for $\beta_{i}=0$ the relation $\left(\xi^{(i)}\right)^{\beta_{i}}=1$ is true.

We denote by $\pi_{m}$ the linear space of polynomials of degree not above $m$. This space is invariant for affin transformations of the space $\mathbf{R}^{\mathbf{m + 1}}$.

Lemma 2. Let $f$ be a linear functional, given over the space of all polynomials of degree at most $m$, let $\left\{\mathbf{e}_{i}\right\}_{i=1}^{n}$ and $\left\{\mathbf{l}_{i}\right\}_{i=1}^{n}$ be the two bases in space $\mathbf{R}^{\mathbf{n}}$. Let $x$ be a vector in this space, $x=x^{(1)} \mathbf{e}_{1}+\ldots+$ $x^{(n)} \mathbf{e}_{n}$. If $A$ is a non-singular linear transformation of coordinates, $y=A x, y=y^{(1)} \mathbf{l}_{1}+\ldots+y^{(n)} \mathbf{l}_{n}$., then the relations

$$
\begin{gathered}
f\left(\left(x^{(1)}\right)^{\alpha_{1}} \ldots\left(x^{(n)}\right)^{\alpha_{n}}\right)=0 \\
\forall \alpha=\left(\alpha_{1}, \ldots, \alpha_{n}\right), \quad|\alpha|=m,
\end{gathered}
$$

are equivalent to the relations

$$
\begin{gathered}
f\left(\left(y^{(1)}\right)^{\beta_{1}} \ldots\left(y^{(n)}\right)^{\beta_{n}}\right)=0 \\
\forall \beta=\left(\beta_{1}, \ldots, \beta_{n}\right), \quad|\beta|=m, y \in \mathbf{R}^{\mathbf{n}} .
\end{gathered}
$$

Proof. It is evident that the set $Q_{m}(x)$ of polynomials of degree $m$ is linear space

$$
Q_{m}=\left\{P_{m} \mid P_{m}(x)=\sum_{|\alpha|=m} p_{\alpha} x^{\alpha}\right\} .
$$

A linear non-singular transformation of variables induces such the transformation of the space $Q_{m}$ at which the degree polynomials do not change. Thus, the space $Q_{m}$ is invariant under the specifie transformation. Therefore, the functional $f$ preserves zero on all space elements $Q_{m}$. Thus, formulas (10) and (11) are equivalent. This completes the proof.

\subsection{Modificatio of atlas. Functions on the manifold}

Let $q$ be some constant, $q \in(0,1)$. We replace the initial atlas $\left\{\psi_{\zeta}: E_{\zeta} \mapsto U_{\zeta} \mid \zeta \in \mathbf{M}\right\}$ with a new atlas $\left\{\psi_{\zeta}: E_{\zeta} \mapsto U_{\zeta}^{\prime} \mid \zeta \in \mathbf{M}\right\}$, where $E_{\zeta}^{\prime}$ is an open ball in $\mathbf{R}^{\mathbf{n}}$ centered at zero and the radius at $q$ is smaller than the radius of the ball $E_{\zeta}$, and $U_{\zeta}^{\prime}=\psi_{\zeta}\left(E_{\zeta}^{\prime}\right)$. Multiplication mapping $\psi_{\zeta}^{-1}$ onto a smooth cutoff function build the reflectio $\left[\psi_{\zeta}^{-1}\right]$, which coincides with $\psi_{\zeta}^{-1}$ in $U_{\zeta}^{\prime}$ and equal to zero in $\mathbf{M}_{\zeta} \backslash \mathbf{U}_{\zeta}$. Since the original atlas is no longer used, we will assign its designation to the new one.

Definitio 1. If the family functions $\left\{F_{\zeta}(t)\right\}_{\zeta \in \mathbf{M}}$ exists and has properties

1) $F_{\zeta} \in C^{s}\left(E_{\zeta}\right) \quad \forall \zeta \in \mathbf{M}$,

2) $F_{\zeta}\left(\psi_{\zeta}^{-1}(\xi)\right) \equiv F_{\zeta^{\prime}}\left(\psi_{\zeta^{\prime}}^{-1}(\xi)\right) \quad \forall \xi \in U_{\zeta} \cap U_{\zeta^{\prime}}$ $\forall \zeta, \zeta^{\prime} \in \mathcal{Z}$ for which $U_{\zeta} \cap U_{\zeta^{\prime}} \neq \emptyset$,

then they say that on a differentiable manifold $\mathbf{M}$ function $u(\xi), u \in C^{s}(M)$, is given. This function can be defined by the relations

$$
u(\xi) \equiv F_{\zeta}\left(\psi_{\zeta}^{-1}(\xi)\right) \quad \forall \xi \in U_{\zeta} \quad \forall \zeta \in \mathbf{M} .
$$

\section{Approximation Relations}

\subsection{The Taylor formula on manifold}

Let a function $u$ be given on the manifold $\mathbf{M}, u \in$ $C^{m+1}(\mathbf{M})$. By definitio 1, this means that the functions $F_{\zeta} \in C^{m+1}\left(E_{\zeta}\right), \zeta \in \mathbf{M}$, are given such as the function $u, u=F_{\zeta} \circ \psi_{\zeta}^{-1}$. We expand the function $F_{\zeta}$ by Taylor's formula in a neighborhood of the point 0 with the remainder of $R_{\zeta}(x)$ :

$$
F_{\zeta}(x)=\sum_{|\alpha| \leq m} \frac{1}{\alpha !} D^{\alpha} F_{\zeta}(0) x^{\alpha}+R_{\zeta}(x), \quad x \in E_{\zeta} .
$$

Then

$$
\begin{gathered}
u(\xi)=F_{\zeta}\left(\psi_{\zeta}^{-1}(\xi)\right)= \\
=\sum_{|\alpha| \leq m} \frac{1}{\alpha !} D^{\alpha} F_{\zeta}(0)\left[\psi_{\zeta}^{-1}(\xi)\right]^{\alpha}+R_{\zeta}\left(\psi_{\zeta}^{-1}(\xi)\right), \quad x \in E_{\zeta} .
\end{gathered}
$$

So, in the neighborhood $U_{\zeta}$ of points $\zeta$ the function $u$ acquires the decomposition

$$
u(\xi)=\left(\mathcal{P}_{\zeta} u\right)(\xi)+\left(\mathcal{R}_{\zeta} u\right)(\xi), \quad \xi \in U_{\zeta},
$$

where

$$
\begin{gathered}
\left(\mathcal{P}_{\zeta} u\right)(\xi)=\sum_{|\alpha| \leq m} \frac{1}{\alpha !} D^{\alpha} F_{\zeta}(0)\left[\psi_{\zeta}^{-1}(\xi)\right]^{\alpha}, \\
\left(\mathcal{R}_{\zeta} u\right)(\xi)=R_{\zeta}\left(\psi_{\zeta}^{-1}(\xi)\right)
\end{gathered}
$$




\subsection{Approximation functional}

Let $f(\eta)$ be an abstract function $\eta \in \mathbf{M}$ with values in the space $(C(\mathbf{M}))^{*}$. Thus if $\eta$ is a fi ed point on $\mathbf{M}$ then $f(\eta)$ is a linear functional over $C(\mathbf{M})$.

Let the support of the functional $f(\eta)$ (for fi ed $\eta)$ lies in the set $U_{\eta}$. Using the representation (15) (16) in $U_{\zeta}$, we get

$$
(f(\zeta), u)=\left(f(\zeta), \mathcal{P}_{\zeta} u\right)+\left(f(\zeta), \mathcal{R}_{\zeta} u\right), \quad \zeta \in \mathbf{M} .
$$

We suppose that

$$
\left(f(\zeta), \mathcal{P}_{\zeta} u\right) \equiv u(\zeta) \quad \forall u \in C^{m+1}(\mathbf{M}) .
$$

A functional $f(\zeta)$ with property (18) is called approximation functional.

\subsection{Approximation relations}

The function

$$
\widetilde{u}(\zeta)=(f(\zeta), u)
$$

is called an approximation of the function $u$. By formulas (17) - (19) we have

$$
u(\zeta)-\widetilde{u}(\zeta)=\left(f(\zeta), \mathcal{R}_{\zeta} u\right)
$$

Lemma 3. For relation (18) to be fulfilled in the class $C^{m+1}$ it is necessary and sufficient to have

$$
\left(f(\zeta),\left[\psi_{\zeta}^{-1}(\bullet)\right]^{\alpha}\right)=\delta_{0, \alpha}, \quad|\alpha| \leq m .
$$

The sign • denotes a variable relative to which the functional $f(\zeta)$ acts, and $\delta_{\alpha, \beta}$ denotes the Kronecker symbol.

Proof. If relation (2.6) is right then we have

$$
\left(f(\zeta), \mathcal{P}_{\zeta} u\right)=\sum_{|\alpha| \leq m} \frac{1}{\alpha !} D^{\alpha} F_{\zeta}(0)\left(f(\zeta),\left[\psi_{\zeta}^{-1}(\bullet)\right]^{\alpha}\right)=F_{\zeta}(0) .
$$

By (2) and (12) we obtain $F_{\zeta}(0)=u(\zeta)$. Therefore relation (18) is true. The reversibility of the reasoning is obvious. This ends the proof.

Identities (21) are called the approximation relations.

Remark 1. If $\mathbf{M} \subset \mathbf{R}^{\mathbf{n}+\mathbf{1}}$, and

$$
\psi_{\zeta}^{-1}(\xi)=\xi-\zeta+o(\|\xi-\zeta\|)
$$

hold then for a small enough diameter of the set $\operatorname{supp} f(\zeta) \cup\{\zeta\}$ relations (21) are close to similar relations for the plane (i.e. in the case when $\mathbf{M}=\mathbf{R}^{\mathbf{n}}$ ):

$$
\left(f(\zeta),(\bullet-\zeta)^{\alpha}\right)=\delta_{0, \alpha}, \quad|\alpha| \leq m .
$$

where $f_{j} \in\left(C^{m+1}\right)^{*}$. In this case, conditions (21)

\subsection{Multiplicity of covering}

Before turning to a more specifi situation, we introduce some definitions

Let $\mathbf{M}$ contain a family of subsets $\left\{M_{j}\right\}_{j \in J}$, $\left\{M_{j}\right\}_{j \in J}=\left\{M_{j} \mid M_{j} \subset \mathbf{M}, \mathbf{j} \in \mathbf{J}\right\}$. We denote by $M_{j}^{\prime}{ }_{j}$ the largest open set, lying in $M_{j}$. Consider the set $\widetilde{\mathbf{M}}=\mathbf{M} \backslash\left(\cup_{\mathbf{j} \in \mathbf{J}} \mathbf{M}_{\mathbf{j}} \backslash \mathbf{M}_{\mathbf{j}}^{\prime}\right)$.

For points $t \in \widetilde{\mathbf{M}}$ the concept of multiplicity of a covering by the family $\left\{M_{j}\right\}$ is introduced in the following way.

Definitio 2. Let $t \in \widetilde{\mathbf{M}}$. The multiplicity $\kappa^{(t)}\left(\left\{M_{j}\right\}\right)$ of the covering of the point $t$ with the family $\left\{M_{j}\right\}$ is the number of sets $M_{j}^{\prime}$ containing point $t$. If the multiplicity of the covering of any point $t$ from the set $t \in \widetilde{\mathbf{M}}$ is the same,

$$
k=\kappa^{(t)}\left(\left\{M_{j}\right\}\right) \quad \forall t \in \widetilde{\mathbf{M}},
$$

then the family $\left\{M_{j}\right\}$ is called the $k$-fold covering for M.

Note that a $k$-fold cover may not be a cover for $\mathbf{M}$ in the usual sense of the word, since the union $\cup_{j \in J} M_{j}$ does not necessarily contain the manifold M.

\subsection{Multiplicity of the family}

Definitio 3. Let $\left\{\omega_{j}\right\}_{j \in J}$ be a family of functions defined on the manifold $\mathbf{M}$. If the set of supports of these functions forms a $k$-fold covering for $\mathbf{M}$, then the number $k$ is called the multiplicity of the family $\left\{\omega_{j}\right\}_{j \in J}$ for $\mathbf{M}$.

As an example, consider the $\left\{\omega_{j}\right\}$ family with fi nite multiplicity for $\mathbf{M}$. Let

$$
f(\zeta)=\sum_{j} \omega_{j}(\zeta) f_{j},
$$
takes the view

$$
\sum_{j} \omega_{j}(\zeta)\left(f_{j},\left[\psi_{\zeta}^{-1}(\bullet)\right]^{\alpha}\right)=\delta_{0, \alpha}, \quad|\alpha| \leq m .
$$

In particular, let $\left\{\xi_{j}\right\}$ be the grid on M. Let $f_{j}=\delta_{\xi_{j}}$ be $\delta$-function at $\xi_{j}$ ). Then relations (24) - (25) can be rewritten as

$$
\sum_{j} \omega_{j}(\zeta)\left[\psi_{\zeta}^{-1}\left(\xi_{j}\right)\right]^{\alpha}=\delta_{0, \alpha}, \quad|\alpha| \leq m .
$$

In the case when $\mathbf{M}=\mathbf{R}^{\mathbf{n}}$, we have an analog of relation (23),

$$
\sum_{j} \omega_{j}(\zeta)\left(\xi_{j}-\zeta\right)^{\alpha}=\delta_{0, \alpha}, \quad|\alpha| \leq m .
$$


Consider another example of approximation relations. Let $\mathbf{M}$ be $n$-dimensional sphere of radius $r$. For orthogonal projection (6) onto the tangent plane, we obtain approximation relations in the form

$$
\sum_{j} \omega_{j}(\zeta)\left(\xi_{j}-\left(\xi_{j}, \zeta\right) \zeta r^{-2}\right)^{\alpha}=\delta_{0, \alpha}, \quad|\alpha| \leq m .
$$

Note that under the condition $r \rightarrow+\infty, \xi_{j} \rightarrow \xi_{j}^{*}$, we obtain analogue relations (28) go over into relations obtained from (27) by replacing $\xi_{j}$ to $\xi_{j}^{*}$.

Of particular interest is the operator

$$
f(\zeta) u=\int_{\mathbf{M}} \mathcal{K}(\zeta, \xi) u(\xi) d \xi
$$

with kernel $\mathcal{K}(\zeta, \xi)$ centered around the diagonal, $\operatorname{supp}_{\xi} \mathcal{K}(\zeta, \xi) \subset U_{\zeta}$ (the index $\xi$ means that the support is define with respect to the variable $\xi$ ). Such an operator is called operator of generalized averaging. If condition (21) is satisfied

$\int_{\mathbf{M}} \mathcal{K}(\zeta, \xi)\left[\psi_{\zeta}^{-1}(\xi)\right]^{\alpha} d \xi=\delta_{0, \alpha}, \quad|\alpha| \leq m, \quad \zeta \in \mathbf{M}$,

then the generalized approximation operator is said to have rank $m$.

\section{On Construction of the Approxi- mations}

\subsection{Approaches to constructing an approxi- mation}

Here we consider two approaches to constructing the approximation of functions, given on the manifold (see also [1] - [5])). The firs approach is the direct use of the above approximation relations for the discussed manifold, the extraction of these coordinate functions and the constructing approximations by the elements corresponding to the linear space. The second approach is related to using the atlas to connect a well-designed approximation apparatus on the plane (finit element approximation, etc.).

Both approaches require no processes cutting the manifold into a finit number of parts and then gluing the approximations obtained on each of the mentioned parts.

The firs approach is characterized by the independent construction and direct solution of the approximation relations (25). In this case the approximation relations (25) are considered as a system of linear algebraic equations (with respect to unknowns $\omega_{j}(\zeta)$ ). This approach is called direct approximation construction.
In the second approach, an approximation on a manifold is induced by the approximations in tangent spaces (for example, Courant or the Zlamal or the Argyris fla approximations, etc.) In complex cases (for $m \geq 2$ or for increased requirements for smoothness) the second approach is more convenient.

\subsection{Direct approximation construction}

In the firs approach, the problem is to fin a solution to the system (25). The number of scalar equations of this system is $M_{m}=\frac{(M+N) !}{m ! N !}$. Striving for the simplest option (namely, to the consideration of the system of linear algebraic equations with a nonsingular square matrix), we require that 1) the multiplicity covering of the system $\left\{\omega_{j}\right\}$ is equal to $M_{m}, 2$ ) the determinant the resulting system was nonzero. The firs of these conditions is determined by the given structure of the supports of the sought functions $\omega_{j}(\zeta)$, and the second condition is ensured by the smallness of these supports.

If the supports are small, then relation (22) begins to play the a certain role. In this case, system (25) is close to system (27). The last one can be rewritten as

$$
\sum_{j \in J(\zeta)} \omega_{j}(\zeta) \xi_{j}^{\alpha}=\zeta^{\alpha}, \quad|\alpha| \leq m,
$$

where $J(\zeta)=\left\{j \mid \zeta \in \operatorname{supp} \omega_{j}\right\}$, and the number of elements $|J(\zeta)|$ of the set $J(\zeta)$ equals the number of $M_{m}$ equations of the system under consideration. In a one-dimensional case, this is a uniquely solvable system with the Vandermonde matrix.

\subsection{Direct approximation of the Courant type}

As an illustration, we construct an analogue of the Courant approximation in the case when the manifold $\mathbf{M}$ is $n$-dimensional sphere. Let $\mathcal{T}$ be a simplicial subdivision of the sphere define by equation (3) in the space $\mathbf{R}^{\mathbf{n}+\mathbf{1}}$. Let $\mathcal{T}^{s}$ denote the $s$-dimensional skeleton of the subdivision $\mathcal{T}$.

In particular, the set $\left\{\xi_{j}\right\}_{j \in J}$ of all its vertices is its zero-dimensional skeleton $\mathcal{T}^{0}, \mathcal{T}^{0}=\left\{\xi_{j}\right\}_{j \in J}$; here $J$ is the set of indices of all vertices of the complex $\mathcal{T}$. For definitenes (without loss of generality), we will assume that the one-dimensional skeleton $\mathcal{T}^{1}$ consists of segments of the shortest paths on the considered area. Without going into the standard description structure of a simplicial complex, we note only that elements of the $n$-dimensional skeleton are considered open $n$-dimensional simplices (i.e., the set of interior points of a closed ordinary $n$-dimensional 
simplex). These simplices are denoted by the letter $T$, and by $\bar{T}$ the closure of the simplex $T$.

The body $\mathcal{Z}_{j}$ of the barycentric star for the vertex $\xi_{j}$ is the union of all closed simplices containing vertex $\xi_{j}$,

$$
\mathcal{Z}_{j}=\bigcup_{\xi_{j} \in \bar{T}} \overline{\forall T \in \mathcal{T}^{n}} \text {. }
$$

The subdivision $\mathcal{T}$ is considered so small that each of its $n$-dimensional simplex is uniquely mapped (by orthogonal projection) to at least on one $n$ dimensional coordinate plane in $\mathbf{R}^{\mathbf{n}+\mathbf{1}}$. In addition, we assume that the number of $n$-dimensional simplices $\mathcal{T}$ is greater than $n+1$.

Consider the system of equations (28) for $m=$ 1. The number of equations in this system is equal to $n+1$, and there are infinitel many solutions of the system. Let us select from them the solution that satisfie the condition

$$
\omega_{j}(\zeta) \equiv 0 \quad \text { for } \quad \zeta \in T \wedge \xi_{j} \notin \bar{T} \quad \forall j \in J .
$$

Theorem 1. If $m=1$ and the condition $(A)$ is fulfilled then the solution of equations (28) has a form

$$
\omega_{j}(\zeta)=\Delta_{j}^{(T)} / \Delta_{*}^{(T)}, \quad j=1,2, \ldots, n, \quad \zeta \in T,
$$

where $\Delta_{j}^{(T)}$ and $\Delta_{*}^{(T)}$ are determinants. The first of them is obtained from the determinant $\Delta^{(T)}$,

$$
\Delta^{(T)}=\operatorname{det}\left(\begin{array}{ccc}
\xi_{1}^{(1)} & \ldots & \xi_{n+1}^{(1)} \\
\ldots & \ldots & \ldots \\
\xi_{1}^{(n+1)} & \ldots & \xi_{n+1}^{(n+1)}
\end{array}\right)
$$

by replacing $j$-th column with the column $\left(\zeta^{(1)}, \ldots, \zeta^{(n+1)}\right)^{T}$. The determinant in the denominator of relation (30) has the form

$$
\begin{gathered}
\Delta_{*}^{(T)}= \\
\operatorname{det}\left(\begin{array}{cccc}
\xi_{1}^{(1)}-\xi_{n+1}^{(1)} & \ldots & \xi_{n}^{(1)}-\xi_{n+1}^{(1)} & \zeta^{(1)} \\
\xi_{1}^{(n+1)}-\xi_{n+1}^{(1)} & \cdots & \xi_{n}^{(n+1)}-\xi_{n+1}^{(n+1)} & \zeta^{(n+1)}
\end{array}\right) .
\end{gathered}
$$

Proof. Condition $(A)$ means that the function $\omega_{j}(\zeta)$ is equal to zero outside of the barycentric star for the vertices $\xi_{j}$. Therefore fixin the point $\zeta$ in some simplex $T$, and using (28), we obtain a system of linear algebraic equations with a square matrix of $n+1$-th order. Let the vertices of the simplex $T$ be points $\xi_{1}, \xi_{2}, \ldots, \xi_{n}, \xi_{n+1}$. By hypothesis, this simplex is uniquely projected onto at least one of the $n$ dimensional coordinate planes of the space $\mathbf{R}^{\mathbf{n}+\mathbf{1}}$. Let such a plane be the plane $\xi^{n+1}=0$. Then the matrix of the system can be written as

$$
\left(\begin{array}{ccc}
1 & \ldots & 1 \\
\xi_{1}^{(1)}-\zeta^{(1)} r^{-2}\left(\xi_{1}, \zeta\right) & \ldots & \xi_{n+1}^{(1)}-\zeta^{(1)} r^{-2}\left(\xi_{n+1}, \zeta\right) \\
\xi_{1}^{(n)}-\zeta^{(n)} r^{-2}\left(\xi_{1}, \zeta\right) & \ldots & \xi_{n+1}^{(n)}-\zeta^{(n)} r^{-2}\left(\xi_{n+1}, \zeta\right)
\end{array}\right)
$$

Simple transformations of the determinant of this matrix lead to the determinant $\Delta_{*}^{(T)}$.

We give a proof in the case $n=2$. For $n>2$ the proof is similar.

In the case $n=2$, the matrix of the system (28) has the form

$$
\left(\begin{array}{ccc}
\xi_{1}^{(1)}-\zeta^{1)} r^{-2}\left(\xi_{1}, \zeta\right) & \xi_{2}^{(1)}-\zeta^{(1)} r^{-2}\left(\xi_{2}, \zeta\right) & \xi_{3}^{(1)}-\zeta^{(1)} r^{-2}\left(\xi_{3}, \zeta\right) \\
\xi_{1}^{(2)}-\zeta^{(2)} r^{-2}\left(\xi_{1}, \zeta\right) & \xi_{2}^{(2)}-\zeta^{(2)} r^{-2}\left(\xi_{2}, \zeta\right) & \xi_{3}^{(2)}-\zeta^{(2)} r^{-2}\left(\xi_{3}, \zeta\right)
\end{array}\right) .
$$

To fin $\omega_{3}(\zeta)$ using Cramer's formula, replace the last column in the determinant of the system under consideration to the column on the right-hand side of the system, namely, to column $(1,0,0)^{T}$. As a result, we get

$$
\begin{gathered}
\Delta_{3}= \\
=\operatorname{det}\left(\begin{array}{ccc}
1 & 1 \\
\xi_{1}^{(1)}-\zeta^{(1)} r^{-2}\left(\xi_{1}, \zeta\right) & \xi_{2}^{(1)}-\zeta^{(1)} r^{-2}\left(\xi_{2}, \zeta\right) & 0 \\
\xi_{1}^{(2)}-\zeta^{(2)} r^{-2}\left(\xi_{1}, \zeta\right) & \xi_{2}^{(2)}-\zeta^{(2)} r^{-2}\left(\xi_{2}, \zeta\right) & 0
\end{array}\right) .
\end{gathered}
$$

Thus

$$
\begin{gathered}
\Delta_{3}= \\
=\operatorname{det}\left(\begin{array}{cc}
\xi_{1}^{(1)}-\zeta^{(1)} r^{-2}\left(\xi_{1}, \zeta\right) & \xi_{2}^{(1)}-\zeta^{(1)} r^{-2}\left(\xi_{2}, \zeta\right) \\
\xi_{1}^{(2)}-\zeta^{(2)} r^{-2}\left(\xi_{1}, \zeta\right) & \xi_{2}^{(2)}-\zeta^{(2)} r^{-2}\left(\xi_{2}, \zeta\right)
\end{array}\right) .
\end{gathered}
$$

Obviously, $\Delta_{3}$ can be represented as

$$
\begin{gathered}
\Delta_{3}= \\
=\operatorname{det}\left(\begin{array}{ccc}
\left(\xi_{1}, \zeta\right) & \left(\xi_{2}, \zeta\right) & 1 \\
\xi_{1}^{(1)}-\zeta^{(1)} r^{-2}\left(\xi_{1}, \zeta\right) & \xi_{2}^{(1)}-\zeta^{(1)} r^{-2}\left(\xi_{2}, \zeta\right) & 0 \\
\xi_{1}^{(2)}-\zeta^{(2)} r^{-2}\left(\xi_{1}, \zeta\right) & \xi_{2}^{(2)}-\zeta^{(2)} r^{-2}\left(\xi_{2}, \zeta\right) & 0
\end{array}\right) .
\end{gathered}
$$

Multiplying the firs line by $r^{-2} \zeta^{(1)}$ and adding result to second line and then multiplying the firs line by $r^{-2} \zeta^{(2)}$ and adding result to the third line, we get

$$
\Delta_{3}=\operatorname{det}\left(\begin{array}{ccc}
\left(\xi_{1}, \zeta\right) & \left(\xi_{2}, \zeta\right) & 1 \\
\xi_{1}^{(1)} & \xi_{2}^{(1)} & r^{-2} \zeta^{(1)} \\
\xi_{1}^{(2)} & \xi_{2}^{(2)} & r^{-2} \zeta^{(2)}
\end{array}\right) .
$$

After obvious transformations, we fin

$$
\begin{gathered}
\Delta_{3}=\left(\zeta^{(1)} \zeta^{(2)}\right)^{-1} r^{-2} \times \\
\times \operatorname{det}\left(\begin{array}{ccc}
\left(\xi_{1}, \zeta\right) & \left(\xi_{2}, \zeta\right) & r^{2} \\
\xi_{1}^{(1)} \zeta^{(1)} & \xi_{2}^{(1)} \zeta^{(1)} & {\left[\zeta^{(1)}\right]^{2}} \\
\xi_{1}^{(2)} \zeta^{(2)} & \xi_{2}^{(2)} \zeta^{(2)} & {\left[\zeta^{(2)}\right]^{2}}
\end{array}\right) .
\end{gathered}
$$

Let's take into account that the firs line contains scalar products. Subtracting the second and third lines from the firs line, and the relation $r^{2}-\left[\zeta^{(1)}\right]^{2}-$ $\left[\zeta^{(2)}\right]^{2}=\left[\zeta^{(3)}\right]^{2}$. As a result we get

$$
\Delta_{3}=\left(\zeta^{(1)} \zeta^{(2)}\right)^{-1} r^{-2} \times
$$

$$
\times \operatorname{det}\left(\begin{array}{lll}
\xi_{1}^{(3)} \zeta^{(3)} & \xi_{2}^{(3)} \zeta^{(3)} & {\left[\zeta^{(3)}\right]^{2}} \\
\xi_{1}^{(1)} \zeta^{(1)} & \xi_{2}^{(1)} \zeta^{(1)} & {\left[\zeta^{(1)}\right]^{2}} \\
\xi_{1}^{(2)} \zeta^{(2)} & \xi_{2}^{(2)} \zeta^{(2)} & {\left[\zeta^{(2)}\right]^{2}}
\end{array}\right)=
$$




$$
=\zeta^{(3)} r^{-2} \operatorname{det}\left(\begin{array}{lll}
\xi_{1}^{(3)} & \xi_{2}^{(3)} & \zeta^{(3)} \\
\xi_{1}^{(1)} & \xi_{2}^{(1)} & \zeta^{(1)} \\
\xi_{1}^{(2)} & \xi_{2}^{(2)} & \zeta^{(2)}
\end{array}\right)
$$

Thus $\Delta_{3}(\zeta)=\zeta^{(3)} r^{-2} \Delta_{3}^{0}(\zeta)$, where

$$
\Delta_{3}^{0}(\zeta)=\operatorname{det}\left(\begin{array}{lll}
\xi_{1}^{(1)} & \xi_{2}^{(1)} & \zeta^{(1)} \\
\xi_{1}^{(2)} & \xi_{2}^{(2)} & \zeta^{(2)} \\
\xi_{1}^{(3)} & \xi_{2}^{(3)} & \zeta^{(3)}
\end{array}\right)
$$

Analogously we get $\Delta_{j}(\zeta)=\zeta^{(3)} r^{-2} \Delta_{j}^{0}(\zeta)$, $j=1,2$, where

$$
\begin{aligned}
& \Delta_{1}^{0}(\zeta)=\operatorname{det}\left(\begin{array}{lll}
\zeta^{(1)} & \xi_{2}^{(1)} & \xi_{3}^{(1)} \\
\zeta^{(2)} & \xi_{2}^{(2)} & \xi_{3}^{(2)} \\
\zeta^{(3)} & \xi_{2}^{(3)} & \xi_{3}^{(3)}
\end{array}\right), \\
& \Delta_{2}^{0}(\zeta)=\operatorname{det}\left(\begin{array}{lll}
\xi_{1}^{(1)} & \zeta^{(1)} & \xi_{3}^{(1)} \\
\xi_{1}^{(2)} & \zeta^{(2)} & \xi_{3}^{(2)} \\
\xi_{1}^{(3)} & \zeta^{(3)} & \xi_{3}^{(3)}
\end{array}\right) .
\end{aligned}
$$

To calculate the determinant $\Delta(\zeta)$ of the matrix of the system, we use one from the approximation relations, namely

$$
\omega_{1}(\zeta)+\omega_{2}(\zeta)+\omega_{3}(\zeta)=1
$$

Relations $\omega_{j}(\zeta)=\Delta_{j}(\zeta) / \Delta(\zeta), j=1,2,3$, are substituted in the previous identity. As a result we get $\Delta(\zeta)=\zeta^{(3)} r^{-2} \Delta^{0}(\zeta)$, where $\Delta^{0}(\zeta)=\sum_{j=1}^{3} \omega_{j}^{0}(\zeta)$.

Hence, in particular, the relations follow

$$
\omega_{j}(\zeta)=\Delta_{j}^{0}(\zeta) / \Delta^{0}(\zeta), \quad j=1,2,3 .
$$

Note also that determinant (32) for $n=3$ coincides with determinant $\Delta^{0}(\zeta)$. Indeed, in this we have a chain of obvious equalities

$$
\begin{gathered}
\Delta_{*}^{(T)}(\zeta)=\operatorname{det}\left(\xi_{1}-\xi_{3}, \xi_{2}-\xi_{3}, \zeta\right)= \\
=\operatorname{det}\left(\xi_{1}, \xi_{2}-\xi_{3}, \zeta\right)-\operatorname{det}\left(\xi_{3}, \xi_{2}, \zeta\right)= \\
\operatorname{det}\left(\xi_{1}, \xi_{2}, \zeta\right)-\operatorname{det}\left(\xi_{1}, \xi_{3}, \zeta\right)-\operatorname{det}\left(\xi_{3}, \xi_{2}, \zeta\right)= \\
=\Delta_{3}^{0}(\zeta)+\Delta_{2}^{0}(\zeta)+\Delta_{1}^{0}(\zeta)=\Delta^{0}(\zeta) .
\end{gathered}
$$

Note that $\Delta_{*}^{(T)} \neq 0$ for $\zeta \in \bar{T}$. Indeed, in view of the assumption that the simplex $T$ is nondegenerate, the vector $\zeta$ can be uniquely represented in the form $\zeta=\sum_{i=1}^{n+1} b_{i} \xi_{i}$, where $b_{i} \geq 0$. Therefore, assuming a linear dependence of the vectors $\xi_{1}-\xi_{n+1}, \ldots, \xi_{n}-$ $\xi_{n+1}, \zeta$, we come to the conclusion that $\zeta$ can be written in the form $\zeta=\sum_{i=1}^{n} a_{i}\left(\xi_{i}-\xi_{n+1}\right)$. By the mentioned uniqueness for $\zeta$ we have the equalities $a_{i}=b_{i}$, $i=1, \ldots, n, b_{n+1}=-\sum_{i=1}^{n} a_{i}=-\sum_{i=1}^{n} b_{i}$. They are possible only in the case when $b_{k}=0, k=$
$1, \ldots, n+1$. But the last relation is impossible, since $\zeta$ lies on the sphere $\mathbf{M}$, and therefore $\|\zeta\|=r \neq 0$. So it has been proved that $\inf _{\zeta \in T}\left|\Delta_{*}^{(T)}\right|>0$.

Applying Cramer's rule for solving system (28) and using elementary properties of determinants, we obtain formulas (30)-(32). This completes the proof.

It is easy to see that

$$
0<c^{\prime} \rho_{i}^{n} r \leq\left|\Delta_{*}^{(T)}(\zeta)\right| \leq c^{\prime \prime} \rho_{e}^{n} r, \quad \zeta \in T,
$$

where $\rho_{i}$ and $\rho_{e}$ are the radii of the inscribed and circumscribed spheres for an $n$-dimensional rectilinear simplex composed of vectors $\xi_{1}-\xi_{n+1}, \xi_{2}-\xi_{n+1}$, $\ldots, \xi_{n}-\xi_{n+1}$, and $c^{\prime}, c^{\prime \prime}$ are positive constants.

It is easy to see that expression (30) define the function $\omega_{j}(\zeta)$ in those $T$ simplices whose closures have point $\xi_{j}$ as its vertex. According to the condition $(A)$ in the remaining simplices of the subdivision $\mathcal{T}$ this function is zero, so

$$
\operatorname{supp} \omega_{j} \subset \bigcup_{\xi_{j} \in \bar{T}, \forall T \in \mathcal{T}^{n}} \bar{T} .
$$

Thus, the support of the function $\omega_{j}$ is contained in the body barycentric star of vertex $\xi_{j}$.

It is easy to check that the function $\omega_{j}$ is continuous on manifold M. Set $\left\{\omega_{j}\right\}_{\xi_{j} \in \mathbf{M}^{0}}$ is the interpolation basis, since

$$
\omega_{j}\left(\xi_{i}\right)=\delta_{i j}
$$

\subsection{Some remarks in the case $n=1$}

If $n=1$, then $\mathbf{M}$ is a circumference of radius $r$ with center in origin $O$ of coordinate system. We suppose that vectors $\xi_{1}, \xi_{2}, \zeta \in \mathbf{R}^{2}$ have the origin in the point $O$ and their endpoints $A, B, C$ belong to a semi-circumference from $M$ respectively. In this case the expression $\left|\Delta_{*}^{(T)}(\zeta)\right|$ can be written in the form $\left|\operatorname{det}\left(\xi_{1}-\xi_{2}, \zeta\right)\right|$. The last one is the area $S$ of the parallelogram constructed on the vector $\xi_{1}-\xi_{2}$ ? $\zeta$.

In the case under consideration, the (curvilinear) "simplex" $T$ is the arc $\smile A B$ of the circumference. The mentioned "rectilinear simplex" is a chord $\xi_{1}-$ $\xi_{2}=A B$.

Let $A B^{\perp}$ be a straight line passing through the center $O$ of the discussed circumference, and let $A B^{\perp}$ be perpendicular to the chord $A B$. Let $\theta$ be the angle between $A B^{\perp}$ and vector $\zeta=O C$. It is easy to see that the area mentioned above can be calculated by formula $S=\|A B\| r \cos \theta,|\theta|<\alpha / 2$, where $\alpha$ is an angle between vectors $\xi_{1}$ and $\xi_{2}$. Thus, we have $\left|\Delta_{*}^{(T)}(\zeta)\right|=\|A B\| r \cos \theta$. It is clear that the inscribed and circumscribed "circumferences" for "rectilinear simplex" $A B$ are the segment $A B$ itself, 
and therefore $\rho_{i}=\rho_{e}=1 / 2\|A B\|$. So, in the case under consideration, inequality (33) turns into an inequality of the form

$$
\operatorname{cr} \cos (\alpha / 2) \leq\left|\Delta_{*}^{(T)}(\zeta)\right| \leq c r,
$$

where $c=\|A B\|$.

The simplest example of approximation on a onedimensional sphere (circumference) is a piecewise linear interpolation in an angle. This is equivalent to considering a piecewise linear interpolation on a periodic grid in $\mathbf{R}^{\mathbf{1}}$. However, on a sphere with dimensions greater than one, a similar grid is not convenient due to the features of the angular coordinates. This disadvantage does not have interpolation

$$
\widetilde{u}(\zeta)=\sum_{j \in I} u\left(\xi_{j}\right) \omega_{j}(\zeta),
$$

where the functions $\omega_{j}(\zeta)$ are given by formulas (30), (34).

Consider these functions in the one-dimensional case.

We introduce the Cartesian coordinates $(x, y)$ in $\mathbf{R}^{\mathbf{2}}$. Let the manifold $\mathbf{M}$ be the circumference $x^{2}+$ $y^{2}=r^{2}$. Let the points $\xi_{i}=\left(x_{i}, y_{i}\right)$ be points on this circumference, $\xi_{i} \in \mathbf{M}, i=0,1, \ldots, K, K+1$. We will assume that $\xi_{K+1}=\xi_{0}$. If the point $\zeta=(x, y)$ lies on circumference $\mathbf{M}$ between points $\xi_{j}$ and $\xi_{j+1}$, then from (34) we fin

$$
\begin{gathered}
\omega_{j}(\zeta)=\left(y x_{j+1}-x y_{j+1}\right) /\left(\left(x_{j+1}-x_{j}\right) y-\right. \\
\left.-\left(y_{j+1}-y_{j}\right) x\right), \quad \omega_{j+1}(\zeta)= \\
=\left(x y_{j}-y x_{j}\right) /\left(\left(x_{j+1}-x_{j}\right) y-\left(y_{j+1}-y_{j}\right) x\right) .
\end{gathered}
$$

In polar coordinates $x=r \cos \varphi, y=r \sin \varphi$, put $\widetilde{\omega}_{j}(\varphi)=\omega_{j}(r \cos \varphi, r \sin \varphi)$. Then

$$
\widetilde{\omega}_{j}(\varphi)=\left\{\begin{array}{l}
\frac{\sin \left(\varphi_{j-1}-\varphi\right)}{2 \sin \frac{\varphi_{j-1}-\varphi_{j}}{2} \cos \left(\varphi-\frac{\varphi_{j-1}+\varphi_{j}}{2}\right)} \\
\text { for } \varphi^{2}\left(\varphi_{j-1}, \varphi_{j}\right), \\
2 \sin \frac{\varphi_{j}-\varphi_{j+1}\left(\varphi-\varphi_{j+1}\right)}{2} \cos \left(\varphi-\frac{\varphi_{j}+\varphi_{j+1}}{2}\right) \\
\text { for } \varphi^{2} \in\left(\varphi_{j}, \varphi_{j+1}\right), \\
0 \text { for } \varphi \notin\left(\varphi_{j-1}, \varphi_{j+1}\right) .
\end{array}\right.
$$

\subsection{Using approximations in tangent spaces}

The idea behind the second approach is to use fla approximations constructed in tangent spaces.

So, we will assume that we know a certain family of fla approximations. More precisely, suppose that for each $\zeta \in \mathbf{M}$ a family of linear functionals $f_{\zeta}\left(\bar{\xi}^{\prime}\right)$ is given, $\bar{\xi}^{\prime} \in E_{\zeta}, f_{\zeta}\left(\bar{\xi}^{\prime}\right) \in\left(C^{m+1}\left(E_{\zeta}\right)\right)^{*}$ such that

$$
\left(f_{\zeta}\left(\bar{\xi}^{\prime}\right), \varphi_{\alpha}\left(\bullet-\bar{\xi}^{\prime}\right)\right)=\delta_{0, \alpha}
$$

where $|\alpha| \leq m, \quad \bar{\xi}^{\prime} \in E_{\zeta}, \quad \zeta \in \mathbf{M}, \varphi_{\alpha}(x)=x^{\alpha}$, $x \in \mathbf{R}^{\mathbf{n}}$.

Taking into account relation (12) we discuss the representation of the function $u(\xi), u(\xi) \equiv$ $F_{\zeta}\left(\psi_{\zeta}^{-1}(\xi)\right)$. Let us defin a family of functionals $f(\zeta)$ with formula

$$
\begin{gathered}
(f(\zeta), u(\bullet))=\left(f_{\zeta}(0), F_{\zeta}(\bullet)\right) \\
\forall \zeta \in \mathbf{M} \forall \mathbf{u} \in \mathbf{C}^{\mathbf{m + 1}} .
\end{gathered}
$$

The homogeneity and additivity of the functional $f(\zeta)$ are obvious. Assuming the uniform (with respect to $\zeta \in \mathbf{M}$ ) boundedness of the norms of the functionals $f_{\zeta}$, we easily obtain the finit norm of the functional $f(\zeta)$ (uniform with respect to $\zeta \in \mathbf{M}$ ). So in this case $f(\zeta) \in(C(\mathbf{M}))^{*}$.

Theorem 2. The formulas

$$
\begin{gathered}
\left(f(\zeta),\left[\psi_{\zeta}^{-1}(\bullet)\right]^{\alpha}\right)=\left(f_{\zeta}(0), \varphi_{\alpha}(\bullet)\right) \\
\forall|\alpha| \leq m, \\
\left(f_{\zeta}(0), R_{\zeta}(\bullet)\right)=\left(f(\zeta), R_{\zeta}\left(\psi_{\zeta}^{-1}(\bullet)\right)\right)
\end{gathered}
$$

are valid.

Proof. Really we apply the functional $f_{\zeta}(0)$ to both sides of relation (13), and also apply the functional $f(\zeta)$ to the both sides of correlation (14). In accordance with relation (40), the left-hand sides of the obtained ratios are equal. Let's equate the right sides:

$$
\begin{gathered}
\sum_{|\alpha| \leq m} \frac{1}{\alpha !} D^{\alpha} F_{\zeta}(0)\left(f_{\zeta}(0), \varphi_{\alpha}(\bullet)\right)+\left(f_{\zeta}(0), R_{\zeta}(\bullet)\right)= \\
=\sum_{|\alpha| \leq m} \frac{1}{\alpha !} D^{\alpha} F_{\zeta}(0)\left(f(\zeta),\left[\psi_{\zeta}^{-1}(\bullet)\right]^{\alpha}\right)+ \\
+\left(f(\zeta), R_{\zeta}\left(\psi_{\zeta}^{-1}(\bullet)\right)\right) .
\end{gathered}
$$

Note the expressions $\left(f_{\zeta}, R_{\zeta}(x)\right), R_{\zeta}\left(\psi_{\zeta}^{-1}(\xi)\right)$ do not depend on the numbers $D^{\alpha} F_{\zeta}(0)$. Because these numbers can take arbitrary values, we come to equalities (40). Taking into account this result and using identity (42) once more, we obtain formula (41). This completes the proof.

Corollary 1. By (3.9) and (40) it follows that functional $f(\zeta)$, defined with formula (39), satisfies condition (21).

As an example, consider the manifold $\mathbf{M}$ with a sufficientl dense grid on it, $\xi_{j} \in \mathbf{M}, j=1, \ldots, N$. We denote by $J(\zeta)$ the set $\left\{j \mid \xi_{j} \in U_{\zeta}\right\}$ and defin a grid of knots $\xi_{j}^{\prime}(\zeta)=\psi_{\zeta}^{-1}\left(\xi_{j}\right), j \in J(\zeta)$. Let 
$\omega_{\zeta, j}\left(\xi^{\prime}\right)$ be the system of coordinate functions such that for an abstract function

$$
f_{\zeta}\left(\bar{\xi}^{\prime}\right)=\sum_{j \in J(\zeta)} \omega_{\zeta, j}\left(\bar{\xi}^{\prime}\right) \delta_{\xi_{j}^{\prime}}
$$

in some neighborhood $V_{\zeta}$ of zero, $V_{\zeta} \subset E_{\zeta}$, identity (39) is true. In other words, let

$$
\begin{array}{r}
\sum_{j \in J(\zeta)} \omega_{\zeta, j}\left(\bar{\xi}^{\prime}\right)\left(\xi_{j}^{\prime}(\zeta)-\bar{\xi}^{\prime}\right)^{\alpha} \equiv \delta_{0, \alpha} \\
\forall \bar{\xi}^{\prime} \in V_{\zeta} \quad \forall \zeta \in \mathbf{M}, \quad|\alpha| \leq \mathbf{m} .
\end{array}
$$

Definin $f(\zeta)$ with formula (39) - (43), we have

$$
(f(\zeta), u)=\sum_{j \in J(\zeta)} \omega_{\zeta, j}(0) u\left(\xi_{j}\right) .
$$

Taking into account equalities (44) we have

$$
\begin{gathered}
\sum_{j \in J(\zeta)} \omega_{\zeta, j}(0)\left[\psi_{\zeta}^{-1}\left(\xi_{j}\right)\right]^{\alpha} \equiv \delta_{0, \alpha} \\
\forall \zeta \in \mathbf{M},|\alpha| \leq \mathbf{m} .
\end{gathered}
$$

It is seen from identity (46) that if we put

$$
\begin{gathered}
\omega_{j}(\zeta) \equiv \omega_{\zeta, j}(0) \text { for } j \in J(\zeta), \\
\omega_{j}(\zeta) \equiv 0 \text { for } j \notin J(\zeta),
\end{gathered}
$$

then relation (26) holds.

\subsection{Central projection to tangent spaces for sphere}

Let us expand formulas (47) for the case when $\mathbf{M}$ is a sphere. We construct an analogue $\omega_{j}^{0}(\zeta)$ of the function $\omega_{j}(\zeta)$ approximation on the sphere (3) using the central projection on a tangent plane. For the point $\xi \in \mathbf{M}$ we fin $\tau \in \mathbf{R}^{1}$ so that $\tau \xi \in E_{\xi}$, namely put $\tau=r^{2} /(\xi, \xi)$. So, given the choice of start coordinates in $E_{\zeta}$ we have

$$
\begin{aligned}
\psi_{\zeta}^{-1}: \quad \xi \mapsto \xi^{\prime}, \quad \xi^{\prime}=r^{2} \xi /(\xi, \zeta)-\zeta, \\
\psi_{\zeta}: \quad \xi^{\prime} \mapsto \xi, \quad \xi=\left(\xi^{\prime}+\zeta\right) r /\left\|\xi^{\prime}+\zeta\right\| .
\end{aligned}
$$

We put $U_{\zeta}=\left\{\xi \mid(\xi, \zeta) \geq \varepsilon r^{2}, \xi \in \mathbf{M}\right\}$, where $\varepsilon$ is a fi ed number from the interval $(0,1)$.

We assume that the simplicial subdivision of $\mathcal{T}$ is so fin that the closure of the barycentric star for any of the vertices of the simplex $\bar{T}$, containing point $\zeta$, lies in $U_{\zeta}$. The image of the neighborhood $U_{\zeta}$ under the mapping $\psi_{\zeta}^{-1}$ is obviously some ball $E_{\zeta}$ centered at the origin of the plane $L_{\zeta}$.
The advantages of this approach are that one can use the approximation estimates obtained for plane (with the approximations of Courant, Zlamal, Argyris). Using Theorem 2, we obtain the same estimates for these approximations for functions define on the manifold.

Let us illustrate this approach using R. Courant's approximations (with respect analogues of the Zlamal and Argyris approximations on manifold see [1], pp. $52-58$, for details).

Theorem 3. In the case of central projection (48) basic functions (47) transform to the functions $\omega_{j}^{0}(\zeta)$,

$$
\omega_{j}^{0}(\zeta)=\left\{\begin{array}{l}
r^{-2}\left(\xi_{j}, \zeta\right) \Delta_{j}^{T}(\zeta) / \Delta^{T} \\
\quad \text { for } \zeta, \xi_{j} \in \bar{T} \subset\left|\mathcal{Z}_{j}\right|, \\
0 \quad \text { for } \zeta \notin\left|\mathcal{Z}_{j}\right| .
\end{array}\right.
$$

Here $\Delta_{j}^{T}(\zeta)$ and $\Delta^{T}$ are determinants introduced by formula (31).

Proof. Let the set $\Phi_{\zeta}=\left\{T \mid T \in \mathcal{T}, T \subset U_{\zeta}\right\}$ consider with the same incident relations that are in complex $\mathcal{T}$. The resulting complex $\Phi_{\zeta}$ is called a fragment of the complex $\mathcal{T}$ in $U_{\zeta}$.

The image $\Phi_{\zeta}^{\prime}$ of the fragment $\Phi_{\zeta}$ under the discussed map is obviously a complex consisting of rectilinear simplices. Put $\xi_{j}^{\prime}=\xi_{j}^{\prime}(\zeta)=\psi_{j}^{-1} \xi_{j}$ for $\xi_{j} \in \Phi_{\zeta}$ so that

$$
\xi_{j}^{\prime}(\zeta)=r^{2} \xi_{j} /\left(\xi_{j}, \zeta\right)-\zeta .
$$

By assumption in the fragment $\Phi_{\zeta}^{\prime}$, a vertex $\xi_{j}^{\prime}$ exits such that the closure of the body of the barycentric star $\mathcal{Z}_{j}^{\prime}(\zeta)$ contains zero plane $L_{\zeta}$.

Let us build the Courant function $\omega_{\zeta, j}\left(\xi^{\prime}\right)$ corresponding to the vertex $\xi_{j}^{\prime}(\zeta)$. Without loss of generality, we will assume that $\xi^{\prime} \in T^{\prime}=T^{\prime}(\zeta), T^{\prime} \in \mathcal{Z}_{j}^{\prime}$, and the vertices of the simplex $T^{\prime}$ are renumbered $1, \ldots, n+1$, and $j \in\{1, \ldots, n+1\}$. As known,

$$
\omega_{\zeta, j}\left(\xi^{\prime}\right)=\lambda_{j}\left(\xi^{\prime}\right),
$$

where $\lambda_{k}\left(\xi^{\prime}\right)=\lambda_{k, \zeta}\left(\xi^{\prime}\right), k=1, \ldots, n+1$, are denoted barycentric coordinates of the point $\xi^{\prime}$ in the simplex $T^{\prime}(\zeta)$.

By definition the vector $\lambda\left(\xi^{\prime}\right)=$ $\left(\lambda_{1}\left(\xi^{\prime}\right), \ldots, \lambda_{n+1}\left(\xi^{\prime}\right)\right)$ satisfie the equation $A \lambda\left(\xi^{\prime}\right)=e\left(\xi^{\prime}\right)$. Here $A$ is a square matrix $(n+1)$-th order, the firs row of which consists of ones, and the $i$-th line $\xi_{1}^{(i)}, \ldots, \xi_{n+1}^{(i)}$ from $i$-th components vertices of the simplex $T^{\prime}(\zeta)$. In addition, $e\left(\xi^{\prime}\right)$ is column vector $\left(1, \xi^{\prime(1)}, \ldots, \xi^{\prime(n)}\right)$, where $\xi^{\prime(i)}$ is the $i$-th component of the vector $\xi^{\prime}$ in coordinate system of the plane $L_{\zeta}$.

Therefore

$$
\lambda_{i}\left(\xi^{\prime}\right)=\Delta_{T^{\prime}, i}\left(\xi^{\prime}\right) / \Delta_{T^{\prime}},
$$


and $\Delta_{T^{\prime}, i}\left(\xi^{\prime}\right)$ is obtained from the determinant $\Delta_{T^{\prime}}=\operatorname{det} A$ by replacing the $i$-th column with a column $e\left(\xi^{\prime}\right)$. So,

$$
\omega_{\zeta, j}\left(\xi^{\prime}\right)=\left\{\begin{array}{l}
\Delta_{T^{\prime}(\zeta), j}\left(\xi^{\prime}\right) / \Delta_{T^{\prime}(\zeta)} \\
\text { for } \xi^{\prime} \in T^{\prime}(\zeta) \stackrel{\mathcal{Z}^{\prime}}{j} \mid, \\
0 \quad \text { for } \xi^{\prime} \notin\left|\mathcal{Z}_{j}^{\prime}\right| .
\end{array}\right.
$$

From formula (51) - (53) it follows that the function $\omega_{\zeta, j}$ does not depend on the choice of the coordinate system in space $\mathbf{R}^{\mathbf{n}+\mathbf{1}}$.

Using relation (47), we now fin

$$
\omega_{j}^{0}(\zeta)=\left\{\begin{array}{l}
\Delta_{T^{\prime}(\zeta), j}(0) / \Delta_{T^{\prime}(\zeta)} \\
\text { for } \quad \zeta, \xi_{j} \in \bar{T} \\
0 \quad \text { otherwise }
\end{array}\right.
$$

Taking into account the invariance of relations (50) for the transformations of coordinates, we discuss the unit vectors $e_{1}, \ldots, e_{n}$ in $\mathbf{R}^{\mathbf{n}}$ so that they are parallel to the vectors $e_{1}^{\prime}, \ldots, e_{n}^{\prime}$ of the coordinate system selected in $L_{\zeta}$. Besides, we suppose that the unit vector $e_{n+1}$ is directed along the vector $\zeta$. Projecting (50) on the axis $e_{1}, \ldots, e_{n}$, we fin

$$
\xi_{j}^{\prime(k)}=r^{2} \xi_{j}^{(k)} /\left(\xi_{j}, \zeta\right), \quad k=1, \ldots, n .
$$

Substituting (54) into $\Delta_{T^{\prime}}$, we have

$$
\begin{aligned}
& \Delta_{T^{\prime}}=\zeta^{(n+1)} r^{2 n} \prod_{j=1}^{n+1}\left(\xi_{j}, \zeta\right)^{-1} \times \\
& \times \operatorname{det}\left(\begin{array}{ccc}
\xi_{1}^{(n+1)} & \ldots & \xi_{n+1}^{(n+1)} \\
\xi_{1}^{(1)} & \ldots & \xi_{n+1}^{(1)} \\
\ldots & \ldots & \ddot{(n)} \\
\xi_{1}^{(n)} & \ldots & \xi_{n+1}^{(n)}
\end{array}\right) .
\end{aligned}
$$

Similar conversions $\Delta_{T^{\prime}, j}(0)$ give

$$
\begin{gathered}
\Delta_{T^{\prime}, j}(0)=\zeta^{(n+1)} r^{2 n-2} \prod_{\substack{j^{\prime}=1 \\
j^{\prime} \neq j}}^{n+1}\left(\xi_{j^{\prime}}, \zeta\right)^{-1} \times \\
\times \operatorname{det}\left(\begin{array}{ccccc}
\xi_{1}^{(n+1)} & \ldots & \zeta^{(n+1)} & \ldots & \xi_{n+1}^{(n+1)} \\
\xi_{1}^{(1)} & \ldots & \zeta^{(1)} & \ldots & \xi_{n+1}^{(1)} \\
\ldots & \ldots & \ldots & & \\
\xi_{1}^{(n)} & \ldots & \zeta^{(n)} & \ldots & \xi_{n+1}^{(n)}
\end{array}\right) .
\end{gathered}
$$

Thus we obtain relation (49) which is invariant form of $\omega_{j}^{0}(\zeta)$. This concludes the proof.

Corollary 2. The functions $\omega_{j}^{0}(\zeta)$ are continuous and give an interpolation basis on the $\mathcal{T}^{0}$ grid, $\omega_{j}^{0}\left(\xi_{i}\right)=\delta_{i, j}$. Note that these functions satisfy condition (29). The solution of system (26) with mappings (48) under the condition $(A)$ is unique.
Corollary 3. In the case $n=1$ (in the case, when $\mathbf{M}$ is a circumference) we find

$$
\begin{aligned}
& \omega_{j}^{0}(x, y)=\frac{\left(x_{j} x+y_{j} y\right)\left(x y_{j+1}-y x_{j+1}\right)}{r^{2}\left(x_{j} y_{j+1}-y_{j} x_{j+1}\right)}, \\
& \omega_{j+1}^{0}(x, y)=\frac{\left(x_{j+1} x+y_{j+1} y\right)\left(y x_{j}-x y_{j}\right)}{r^{2}\left(x_{j} y_{j+1}-y_{j} x_{j+1}\right)} .
\end{aligned}
$$

Here the point $(x, y)$ lies on the circumference $\mathbf{M}$ between knots $\left(x_{j}, y_{j}\right)$ and $\left(x_{j+1}, y_{j+1}\right)$, namely, on an arc connecting them, on which there are no other grid knots.

Corollary 4. The function

$$
\widetilde{\omega}_{j}^{0}(\varphi)=\omega_{j}^{0}(r \cos \varphi, r \sin \varphi)
$$

represents an interpolating basis of trigonometric splines,

$$
\widetilde{\omega}_{j}^{0}(\varphi)=\left\{\begin{array}{l}
\frac{\cos \left(\varphi_{j}-\varphi\right) \sin \left(\varphi-\varphi_{j-1}\right)}{\sin \left(\varphi_{j}-\varphi_{j-1}\right)} \\
\text { for } \varphi \in\left(\varphi_{j-1}, \varphi_{j}\right], \\
\frac{\cos \left(\varphi_{j}-\varphi\right) \sin \left(\varphi_{j+1}-\varphi\right)}{\sin \left(\varphi_{j+1}-\varphi_{j}\right)} \\
\text { for } \varphi \in\left(\varphi_{j}, \varphi_{j+1}\right] \\
0 \quad \text { for } \varphi \notin\left(\varphi_{j-1}, \varphi_{j+1}\right]
\end{array}\right.
$$

It is clear to see that functions (55) - (56) differ from functions (36) - (37), but all of them have interpolation properties (35) .

\section{Conclusion}

This work is devoted to effective evaluations of the speed of convergence for multidimensional approximations of the functions define on the differential manifold.

We consider two approaches to constructing the approximation of functions, given on the manifold. The firs approach gives opportunity to use the approximation relations for the differentiated manifold. The second approach is related to the use of well-designed approximation apparatus on the plane (finit element approximation, etc.). Both approaches require no processes cutting the manifold into a finit number of parts and then gluing the approximations obtained on each of the mentioned parts.

The firs approach is characterized by the independent construction and direct solution of the approximation relations. In this case the approximation relations are considered as a system of linear algebraic equations (with respect to unknowns $\omega_{j}(\zeta)$ ). This approach is called direct approximation construction.

In the second approach, an approximation on a manifold is induced by the approximations in tangent spaces (for example, the Courant or the Zlamal or the 
Argyris fla approximations, etc.) In complex cases (In the multidimensional case or for increased requirements of smoothness) the second approach is more convenient.

It is assumed that the further development of the obtained results will consist of obtaining of sufficien conditions for the embedding of approximating spaces for functions define on a manifold. The embedding is necessary for a lot of methods that refin the approximate solution, as well as for the wavelet expansions of incoming information.

Acknowledgements: The authors would like to thank RFBR for partly support by Grant N 15-01008817 .

\section{References:}

[1] Yu.K.Dem'yanovich. Local approximation on manifold and minimal splines (monograph). Publishing House of St. Petersburg State University. 1994 (in Russian).

[2] Yu.K.Dem'yanovich. Spline-wavelet decompositions on manifolds//Journal of Mathematical Sciences, 2008. Vol.150, issue 1. Pp.1787-1798

[3] Yu.K.Dem'yanovich. Wavelets on Manifold//Doklady Mathematics. 2009, vol.79, No.1. Pp.'21-24.

[4] Yu.K.Dem'yanovich. Adaptive Haar Type Wavelets on Manifolds//Journal of Mathematical Sciences (United States),251, 6,December 28,2020. Pp. 797-813

[5] Francois Dubeau, Said Elmejdani, Riadh Ksantini. Non-uniform Haar wavelets//Applied Mathematics and Computation. 159, 2004, 675-691. https://www.sciencedirect.com/science/article /abs/pii/S009630030301155X

[6] Novikov I.Ya., Protasov V.Yu., Skopina M.A. Theory splashes), Moscow: FIZMATGIZ, 2005, 616 p. - ISBN 5-9221-0642-2

[7] Saxena, H., Singh, A., Rai, J.N. Adaptive splinebased PLL for synchronisation and power quality improvement in distribution system(2020) IET Generation, Transmission and Distribution, 14 (7), pp. 1311-1319.

[8] Cornel, D., Buttinger-Kreuzhuber, A., Konev, A., Horv?th, Z., Wimmer, M., Heidrich, R., Waser, $\mathrm{J}$. Interactive visualization of floo and heavy rain simulations (2019) Computer Graphics Forum, 38 (3), pp. 25-29.
[9] N. Pervaiz, I. Aziz, Haar wavelet approximation for the solution of cubic nonlinear Schrodinger equations, Physica A: Statistical Mechanics and its Applications, Vol. 545, paper 123738, 2020, DOI: $10.1016 /$ j.physa.2019.123738.

[10] A Raza, A. Khan, Approximate solution of higher order two point boundary value problems using uniform Haar wavelet collocation method, Springer Proceedings in Mathematics and Statistics, 272, 2019, pp. 209-220. DOI: 10.1007/978981-13-9608-3_14.

[11] R. Amin, S. Nazir, I. Garc?a-Magari?o, A collocation method for numerical solution of nonlinear delay integro-differential equations for wireless sensor network and internet of things Sensors (Switzerland),Vol 20,7, paper 1962, 2020.

[12] E.H.S. Diop, A.-O. Boudraa, V.B.S Prasath, Optimal Nonlinear Signal Approximations Based on Piecewise Constant Functions, Circuits, Systems, and Signal Processing, Vol. 39, No 5, 2020, pp. 2673-2694. DOI: 10.1007/s00034-019-01285.

[13] S.Mallat, A Wavelet Tour of Signal Processing, Academic Press, 1999. DOI: 10.2118/96553-MS. [8] Francois Dubeau, Said Elmejdani, Riadh Ksantini, Non-uniform Haar wavelets, Applied Mathematics and Computation, Vol.159, 2004, pp. 675-691, https://www.sciencedirect.com/science/article /abs/pii/S009630030301155X

[14] H, Saxena, A. Singh, J.N. Rai, Adaptive splinebased PLL for synchronisation and power quality improvement in distribution system, IET Generation, Transmission and Distribution, Vol. 14, No 7, 2020, pp. 1311-1319.

[15] D.Cornel, A.Buttinger-Kreuzhuber, A.Konev, Z.Horvath, M.Wimmer, R. Heidrich, J.Waser, Interactive visualization of floo and heavy rain simulations, Computer Graphics Forum, Vol.38, No 3, 2019, pp. 25-29.

[16] Z.Yang, H.Liu, T.Bi, Z.Li, Q.Yang, An adaptive PMU missing data recovery method, International Journal of Electrical Power and Energy Systems, Vol.116, paper 105577, 2020.

[17] I.G.Burova,E.F.Muzafarova,I.I.Narbutovskikh, Local splines of the Second and Third Order, Complex-valued Splines and Image Processing, International Journal of Circuits, Systems and Signal Processing, Vol. 13, 2019, pp. 419-429. 
[18] Yu. K. Dem'yanovich, General Flows and their Adaptive Decompositions, WSEAS Transactions on Mathematics, Volume 17, 2018, pp. 28-34.

[19] B. Sober, D. Levin, Manifold approximation by moving least-squares projection, Constr. Approx., 2019, http://dx.doi.org/10.1007/s00365019-09489-8.

[20] J.A. Costa, A.O. Hero, Geodesic entropic graphs for dimension and entropy estimation in manifold, learning, IEEE Trans. Signal Process. 52 (8), 2004, 2210-2221.

[21] M.-Y. Cheng, H.-t. Wu, Local linear regression on manifolds and its geometric interpretation, $\mathrm{J}$. Amer. Statist. Assoc. 108 (504), 2013, 14211434.

[22] Yu.K. Dem'yanovich, Local approximations on manifolds and weighted estimates, Journal of Soviet Mathematics, 36 (2), 1987 pp. 261-269.

[23] Yu.K.Demyanovich, Spline approximations on manifolds, International Journal of Wavelets, Multiresolution and Information Processing, 4 (3), 2006, pp. 383-403.
[24] Yu.K. Demjanovich, A.V.Zimin, Wavelet decompositions on a manifold, Journal of Mathematical Sciences, 150 (2), 2008, pp. 1929-1936.

[25] Yu.K.Dem'yanovich, Wavelet expansion of functions on a differentiable manifold, Vestnik St. Petersburg University: Mathematics, 41 (4), 2008, pp. 290-297.

[26] Dem'yanovih, Y.K. Embedded spaces and wavelets on a manifold, Journal of Mathematical Sciences, 176 (1), 2011, pp. 7-19.

[27] I. G. Burova, E. F. Muzafarova, Approximations with Polynomial, Trigonometric, Exponential Splines of the Third Order and Boundary Value Problem, International Journal of Circuits, Systems and Signal Processing, Volume 14, 2020, pp. 460-473.

[28] I. G. Burova, Continuous Local Splines of the Fourth Order of Approximation and Boundary Value Problem, International Journal of Circuits, Systems and Signal Processing, Volume 14, 2020 pp. 440-450.

\section{Creative Commons Attribution License 4.0 (Attribution 4.0 International, CC BY 4.0)}

This article is published under the terms of the Creative Commons Attribution License 4.0 https://creativecommons.org/licenses/by/4.0/deed.en_US 